WellBeing International

WBI Studies Repository

9-1991

\title{
Enhanced Attraction to Blood by Pigs with Inadequate Dietary Protein Supplementation
}

\author{
David Fraser \\ Agriculture Canada \\ D. E. Bernon \\ Agriculture Canada \\ R. O. Ball \\ University of Guelph
}

Follow this and additional works at: https://www.wellbeingintlstudiesrepository.org/nutrit

Part of the Animal Studies Commons, Other Animal Sciences Commons, and the Other Nutrition Commons

\section{Recommended Citation}

Fraser, D., Bernon, D. E., \& Ball, R. O. (1991). Enhanced attraction to blood by pigs with inadequate dietary protein supplementation. Canadian journal of animal science, 71(3), 611-619.

This material is brought to you for free and open access by WellBeing International. It has been accepted for inclusion by an authorized administrator of the WBI Studies Repository. For more information, please contact wbisr-info@wellbeingintl.org.

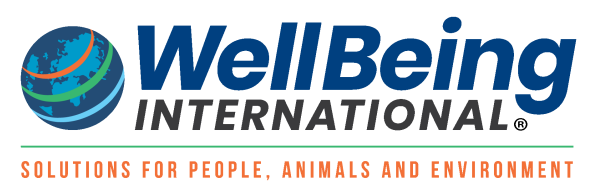




\title{
Enhanced Attraction to Blood by Pigs with Inadequate Dietary Protein Supplementation
}

\author{
David Fraser ${ }^{1}$, D.E. Bernon ${ }^{1}$, and R.O. Ball ${ }^{2}$ \\ ${ }^{1}$ Agriculture Canada \\ ${ }^{2}$ University of Guelph
}

\begin{abstract}
KEYWORDS
pigs, tail-biting, abnormal behavior, amino acids, protein, weight gain, growth
\end{abstract}

\begin{abstract}
In two experiments, 60 individually penned growing pigs were exposed daily to two sections of cotton cord, one of which had been soaked with pigs' blood and subsequently dried, while the other was plain. The animals' preference for chewing-on the bloodimpregnated cord was quantified by direct observation. When fed a standard "control" diet of corn, barley, and soybean meal with mineral and vitamin supplements, the pigs had a clear but modest preference for chewing the blood-impregnated cord. Omission of the protein supplement (soybean meal) from the diet for 4 wk led to a major increase in attraction to blood and a significant reduction in body weight gain. In the second experiment, supplementation of this "negative" diet with (a) synthetic lysine, or (b) synthetic lysine and other synthetic amino acids, led to weight gains that were intermediate between those seen with the control and negative diets. Attraction to blood was also intermediate on average, although not significantly lower than that seen with the negative diet. Pigs on the diets supplemented with amino acids had highly variable weight gains. Those that gained as rapidly as the controls had relatively low attraction to blood, while those showing clear depression of gain tended to (but did not always) have enhanced attraction to blood. It is hypothesized that the depression of growth caused by inadequate protein nutrition predisposed the animals to enhanced attraction to blood, and that such a relationship may help to explain the widely reported link between dietary inadequacies and tail-biting.
\end{abstract}

Inadequate protein supplementation of the diet is often cited as a factor contributing to the behavioral problem of tail-biting by pigs (e.g., Gadd 1967; Jericho and Church 1972; Dalrymple 1978). Such a link could occur for several reasons. First, many of the hormones and brain neurotransmitters associated with regulation of behavior and stress responses of animals are synthesized from amino acids derived from dietary protein (Anderson 1985; Peters and Harper 1985; Anisman and Zacharko 1986; Williamson 1987). Deficiencies, imbalances and excesses of many amino acids and the associated changes in neurotransmitters have been found to alter the animals' behavior (Mench and Shea 1988; Harper and 
Peters 1989) including increased excitability or aggressiveness (Chamberlain et al. 1987; Munoz-Blanco and Castillo 1987) or increased docility (Lacy et al. 1984; Laycock and Ball 1990). In addition, simple dissatisfaction with the diet might increase the animals' level of restlessness and redirected foraging behavior, and hence increase the likelihood of tail injury through excessive rooting and chewing on the bodies of penmates. Finally, a dietary deficiency might cause a specific appetite for nutrients available in blood and thus cause blood to be more attractive.

Previously we have suggested that the attraction to blood shown by some pigs may contribute to an escalation of tail-biting once tail injuries have developed (Fraser 1987a), and that certain dietary mineral deficiencies may exacerbate the problem by causing a heightened attraction to blood (Fraser 1987b). In the following experiments, we studied whether a similar increase in attraction to blood could be induced by feeding pigs on diets with inadequate protein supplementation.

\section{MATERIALS AND METHODS}

Two experiments used 60 Yorkshire and Yorkshire $\times$ Landrace pigs (30 females and 30 castrated males) from the Animal Research Centre's minimum disease herd. Pigs were aged about $75 \mathrm{~d}$ at the beginning of the experiments. About $5 \mathrm{~d}$ before observations began, the animals were moved from large group pens into individual pens measuring $0.91 \times 1.83 \mathrm{~m}$. Eight or ten such pens were used, and the experiments consisted of successive replicates of eight or ten pigs each. Food and water were continuously available. Room temperature was usually at $20-25^{\circ} \mathrm{C}$, with one 16 -h period of light (about $40 \mathrm{Ix}$ ) per day.

The "tail models" consisted of sections of unwaxed white cotton sash cord (Braided Cotton Sash Cord, No. 16, Barry Boulerice Inc., Dollard des Ormeaux, PQ) $18 \mathrm{~cm}$ long and $1.3 \mathrm{~cm}$ in diameter. The gate of each animal's pen was fitted with a clear plastic panel with two holes of $2.5-\mathrm{cm}$ diameter that allowed easy attachment and removal of two tail models $30 \mathrm{~cm}$ apart and $38 \mathrm{~cm}$ above the floor. When attached to the panel, the models could be chewed by the pig in the pen, while an observer watched through the clear plastic. In each observation period the pigs were presented with one plain and one bloodimpregnated model. The latter had been soaked overnight in fresh, whole, pigs' blood (with $400 \mathrm{mg}$ disodium EDTA per litre of blood added as an anticoagulant) and allowed to dry in the air for at least $24 \mathrm{~h}$. Plain tail models were soaked in tap water with the same concentration of anticoagulant and allowed to dry in the air. After being used in one observation period, the models were thoroughly laundered, dried, and resoaked in blood or water (with anticoagulant) before being used again.

On the 3 or $4 \mathrm{~d}$ before observations began, the observer familiarized the pigs with the procedure by allowing them to chew on a tail model in his presence. For the normal daily observation periods, two tail models were attached simultaneously to the plastic panel for 24 min. During this time, a click sounded every $6 \mathrm{~s}$, and the observer noted whether the pig was chewing on either model at the time of the click. Chewing was scored only if the model was inside the pig's mouth; other types of contact with the models were ignored. The observer scored two neighboring pigs simultaneously during observation periods. Tail models were removed at the end of the $24 \mathrm{~min}$. Each pig was observed during one 24-min period per day, $5 \mathrm{~d}$ per week in each week of observations. The animals were tested in the same order each day, so that the time of day for testing an individual was relatively consistent. The positions of the plain and bloodimpregnated models were alternated daily. For each daily 24-min period, each pig was assigned two scores representing the number of observations in which it was chewing (i) the blood-impregnated model, or (ii) the plain model, with a maximum possible daily score of 240 .

Almost all observations were made by the same observer in each experiment. As a check on the reliability of the recording method, 16 of the 24-min periods, selected as convenient, were scored by two 
observers independently. On both measures (chewing the plain model, and chewing the bloodimpregnated model), the scores per 24-min trial were in close agreement for the two observers $(r=0.98)$.

The standard diet (used for all pigs before the experiment began, and as the control diet in both experiments) included $49.9 \%$ corn, $25 \%$ barley, $17 \%$ soybean meal (48\% crude protein), $2 \%$ stabilized fat, and, 1.5\% Ameribond FG calcium lignosulphonate (Daishowa Chemicals, Quebec, Canada) used as a pelleting agent. A vitamin premix was included at $0.5 \%$ and supplied, per kilogram of diet, 8250 IU vitamin $A, 550 \mathrm{IU}$ vitamin $\mathrm{D}_{3}, 27 \mathrm{IU}$ vitamin $\mathrm{E}, 4 \mathrm{mg}$ vitamin $\mathrm{K}, 3 \mathrm{mg}$ thiamine, $8 \mathrm{mg}$ riboflavin, $33 \mathrm{mg}$ pantothenic acid (D form), $44 \mathrm{mg}$ niacin, $500 \mathrm{mg}$ choline, $250 \mu \mathrm{g}$ biotin, and $28 \mu \mathrm{g}$ vitamin $\mathrm{B}_{12}$. Mineral supplements comprised $1.8 \%$ dicalcium phosphate (18\% Ca, 20\% P), 1.3\% limestone (38\% Ca), 0.5\% iodized salt, and $0.5 \%$ trace mineral premix which supplied, per kilogram of diet, $80 \mathrm{mg}$ iron, $75 \mathrm{mg}$ zinc, $20 \mathrm{mg}$ manganese, $8 \mathrm{mg}$ copper, and $0.2 \mathrm{mg}$ selenium.

In both experiments, variation is expressed as standard deviation (SD) where the emphasis is on describing variability, and as standard error of the mean (SEM) where the significance of a difference is of interest.

\section{Experiment 1}

In the first experiment, pigs were fed either the control diet or a "negative" diet from which the soybean meal had been omitted, with the amounts of corn and barley increased proportionately. The objective was to determine whether such a gross inadequacy in protein supplementation would lead to enhanced attraction to blood.

Two like-sexed litter-mates (either two males or two females as convenient) were studied from each of 10 litters, for a total of 20 animals weighing $29.7 \pm 4.5 \mathrm{~kg}$ (mean \pm SD) at the start of the experiment. Ten pigs (from five litters) were tested as one replicate, followed by a second replicate of 10 more.

The experiment lasted 5 wk, with observations made $5 \mathrm{~d}$ per week (Monday to Friday) in each of weeks 0 (pre-test), 2 and 4. During week 0 , all pigs remained on the control diet. On the Friday of week 0 , half the pigs (one from each pair of littermates) were switched to the negative diet for the remaining 4 wk of the experiment, while the other pigs continued to receive the control diet. Because of the large individual differences in response to the tail models, the pig with the higher score for chewing the blood-covered model in week 0 was assigned to the negative diet in half of the littermate pairs, and to the control diet in the other pairs. Pigs were weighed on the Friday of weeks 0 and 4.

Because individual days were not a factor of interest, the daily scores for chewing each type of tail model were averaged to produce an average daily score for each week for each pig. These were used to produce a "preference score" for each pig in each week, calculated as the average daily score for chewing the blood-impregnated model, minus the average daily score for chewing the plain model. Preference scores were analyzed, for each week separately, by analysis of variance using the following factors: diet (1 d.f.), replicates (1 d.f.), and pairs within replicates (8 d.f.), with 9 degrees of freedom for error. Weight gains, calculated during weeks 1-4, were analyzed using a similar model, with initial weight included as a covariate. Sex differences were not of major interest (see Fraser 1987a, b) and were not included in the model because sex was confounded with litter effects; however, the major trends were checked for males and females separately.

\section{Experiment 2}

A simple omission of soybean meal, as in exp. 1, would influence the mineral composition, and likely the flavor and other features of the diet, as well as the levels and ratios of amino acids. The second 
experiment included two deficient diets supplemented with synthetic amino acids. The objective was to determine whether partially restoring the levels of essential amino acids and ideal protein in the negative diet would reduce its impact on attraction to blood.

Four diets were used: (1) the control diet, (2) the negative diet from exp. 1, (3) a "lysine-added" diet, similar to the negative diet but supplemented with synthetic lysine at $4.71 \mathrm{~kg}^{-1}$, calculated to give $7.5 \mathrm{~g}$ lysine per kilogram of diet, and (4) a "supplemented" diet, also similar to the negative diet, but supplemented with synthetic lysine at $4.71 \mathrm{~kg} \mathrm{t}^{-1}$. leucine at 2.81 , isoleucine at 1.17 , methionine at 0.50 , threonine at 1.61 , tryptophan at 0.37 , and valine at $0.30 \mathrm{~kg} \mathrm{t}^{-1}$. These levels in the supplemented diet were intended to give levels of the deficient essential amino acids equivalent to NRC recommendations. However, it was later found that a calculation error had been made, and that leucine should not have been added at all to the supplemented diet. Perhaps because of the resulting imbalance and perhaps for other reasons, the supplemented diet proved to be equivalent to the lysine-added diet in its effect on the animals, weight gains and behavior.

Fig. 1. Preference scores (indicating preference for chewing the blood-impregnated tail model) in weeks 0 (pre-test), 2 and 4, and weight gain over the 4-wk test, for pigs fed the negative (N) or control (C) diets in exp. 1. Preference scores are the average daily score for chewing the blood-impregnated model (maximum of 240), minus the average daily score for chewing the plain model. Each mean \pm SEM is based on $n=10$ pigs per treatment.

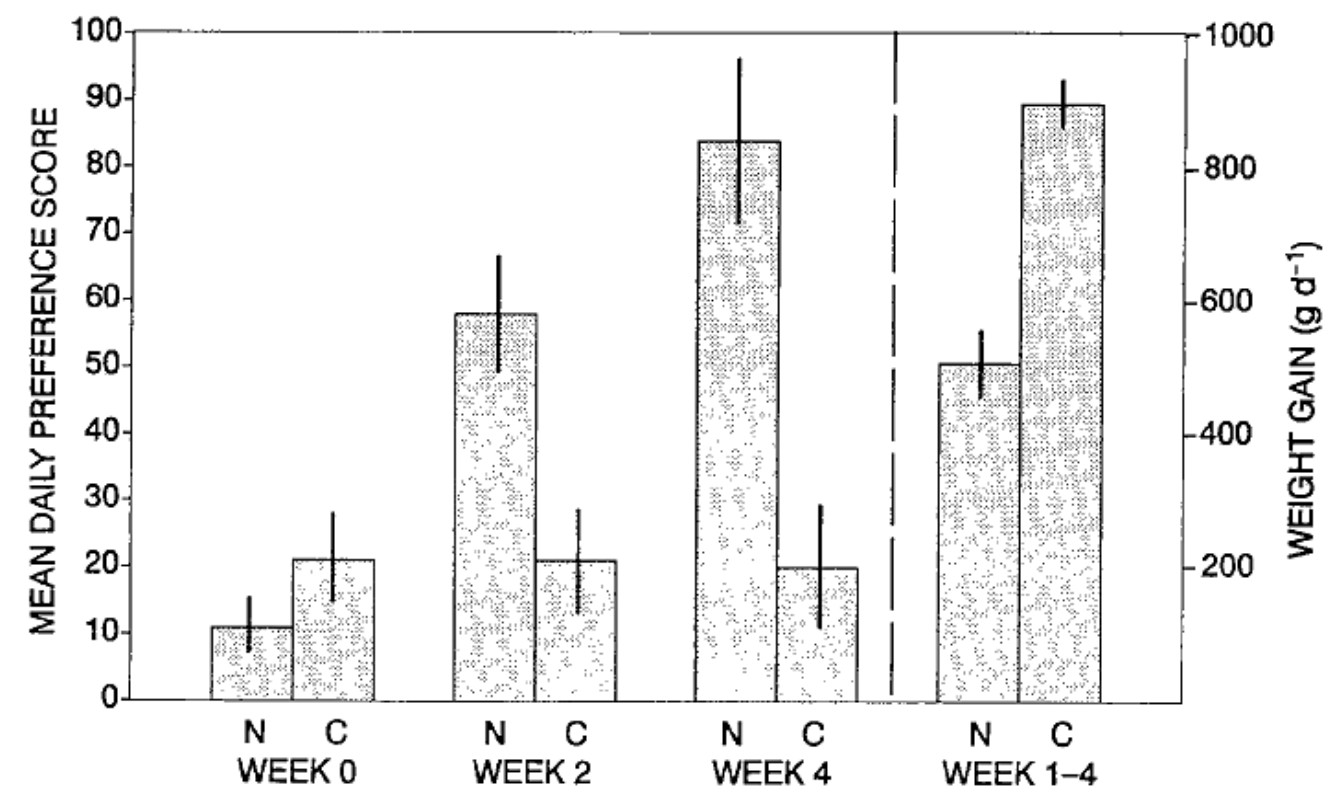

The experiment used 40 pigs: four like-sexed litter-mates from each of 10 litters. Males were used in five litters and females in five, as dictated by availability. Pigs weighed $29.4 \pm 5.6 \mathrm{~kg}$ (mean $\pm \mathrm{SD}$ ) at the beginning of the experiment. Five replicates of two litters (eight pigs) were done in sequence.

As in exp. 1, the pigs were observed in weeks 0 (pre-test), 2 and 4. Pigs were assigned to diets at the end of week 0 by a restricted randomization scheme which ensured that litter-mates with the highest and lowest scores for chewing the blood-impregnated model were assigned to each diet with similar frequency.

Two pigs were omitted from the analysis. One was a control pig which became lame. The other, on the lysine-added diet, failed to adapt to the water nipple. Weekly preference scores for the remaining 38 pigs 
were analyzed for each week separately, by an analysis of variance model that included diet ( 3 d.f.), replicates (4 d.f.), litters within replicates ( 5 d.f.), and error ( 25 d.f.). Sex was not included in the model because it was completely confounded with litter effects, but the major trends were checked for males and females separately. Data on feed intake were collected but not used in the analysis because several animals caused uncontrolled spillage of feed.

Fig. 2. Relation between preference scores in week 4 and body weight gain over the 4-wk test period for the 20 pigs in exp. 1 (a) and the 38 pigs in exp. 2 (b). Each pig's preference score is its average daily score for chewing the blood-impregnated model, minus the average daily score for chewing the plain model. Each point represents one pig on the control (circles), negative (diamonds), lysine-added (triangles), or supplemented (squares) diets.

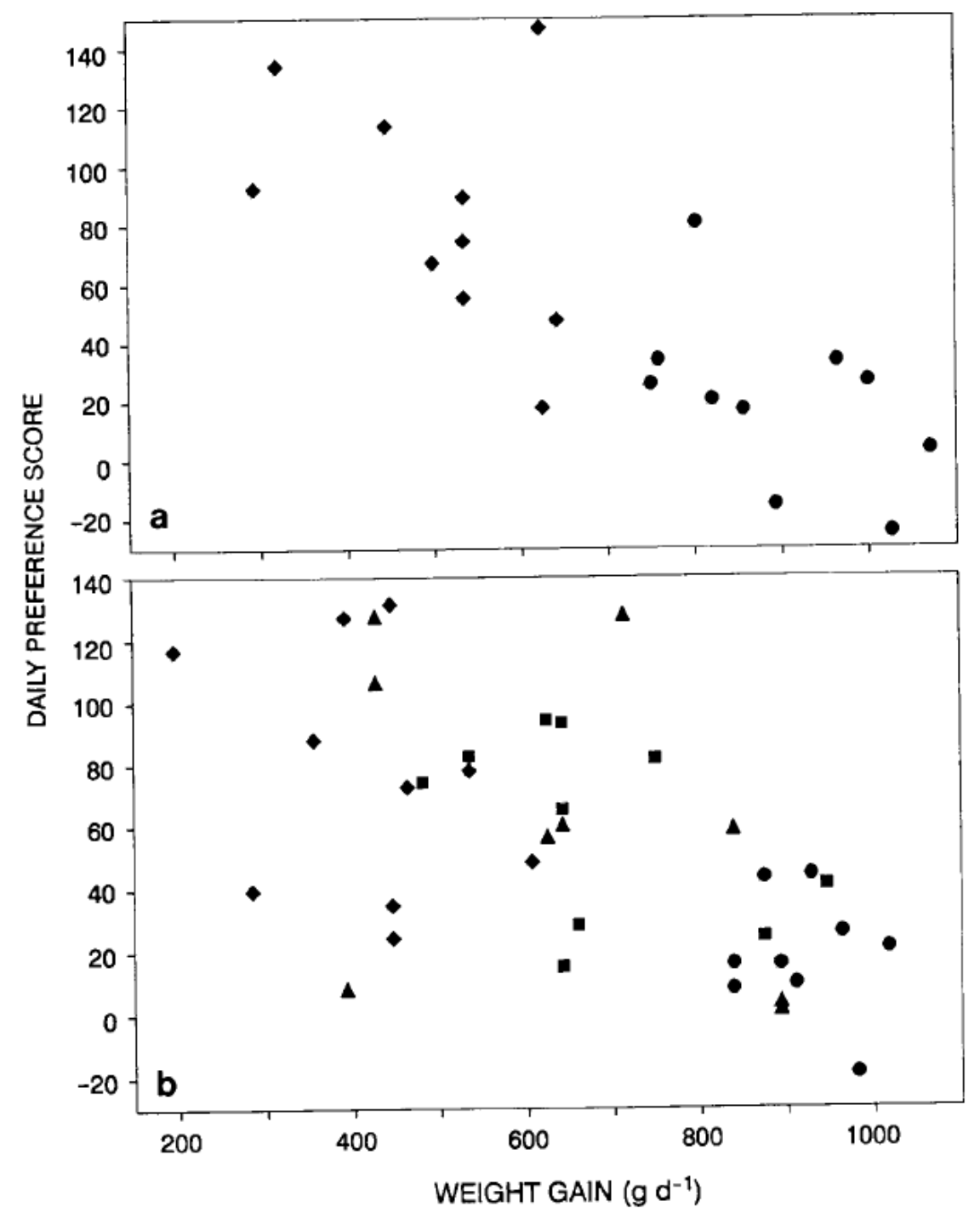

\section{RESULTS}

\section{Experiment 1}

In the pre-test week, 18 of the 20 pigs chewed more on the blood-impregnated model (mean \pm SEM daily score of $52 \pm 4$ ) than on the plain model $(36 \pm 4)$. In subsequent weeks pigs on the control diet showed 
little change in the average amount of chewing they directed at the two models, and they thus maintained a similar level of preference for the blood-covered model throughout the experiment (Fig. 1).

Pigs assigned to the control diet had a somewhat higher average preference score than those on the negative diet in the pre-test (Fig. 1; $P=0.20$ ). By week 2, pigs on the negative diet had approximately doubled their amount of chewing on the blood-impregnated model, and thus had considerably higher preference scores than those on the control diet $(P<0.01)$. The difference was even more pronounced in week $4(P<0.001)$. Weight gains over the $28 \mathrm{~d}$ were much higher on the control $\left(895 \pm 36 \mathrm{~g} \mathrm{~d}^{-1}\right.$, mean \pm SEM) than on the negative diet $\left(506 \pm 38 \mathrm{~g} \mathrm{~d}^{-1}\right.$; Fig. 1; $\left.P<0.001\right)$. Trends were similar for males and females; the females tended to have slightly lower weight gains and slightly higher preference scores, but the sex differences did not approach statistical significance.

Weight gain varied greatly among individuals in a manner that seemed related to the animals' attraction to blood (Fig. 2a). The overall regression of preference score in week 4 on weight gain had a slope of -0.16 \pm 0.031 ( \pm SE). The overall regression is difficult to interpret because it combines within- and betweendiet effects. Therefore, a pooled regression was calculated within diets, by expressing each value as a deviation from the mean of all pigs on the same diet. This gave a similar regression coefficient $(-0.14 \pm$ $0.062 ; P<0.05)$. In view of the relation between weight gain and attraction to blood, the analysis of variance of preference scores was repeated with weight gain included as a covariate. After adjustment for the covariate, the difference attributable to diet became statistically nonsignificant in week $2(P=0.14)$ and week $4(P=0.10)$.

\section{Experiment 2}

In the pre-test week, 36 of the 38 pigs chewed more on the blood-impregnated model (mean \pm SEM of the 38 daily mean scores $=49 \pm 4$ ) than on the plain model $(22 \pm 2)$. On the control diet, chewing on both models tended to decline over the remaining 4 wk (36 \pm 6 and $18 \pm 4$ for the blood-impregnated and plain models respectively in week 4), but the preference for the blood-impregnated model remained clear.

As in exp. 1, the pigs fed the diet lacking soybean meal showed a much stronger attraction to blood than those fed the control diet. The difference was evident in week 2 and very pronounced in week a Fig. 3), and was due to the pigs on the negative diet increasing their chewing on the blood-impregnated model while decreasing their chewing on the control model. The two diets supplemented with amino acids gave an intermediate response on average, but the preference scores did not differ significantly $(P \sim 0.15,1$ tailed, by t-tests for paired comparisons) from those of pigs on the negative diet. Analysis of variance of the preference scores showed significant differences among diets $(P<0.05)$ and among replicates $(P<$ $0.001)$ in week 2 and among diets $(P=0.01)$ in week 4. Duncan's test showed that the control diet differed from all three of the other diets in both weeks 2 and $4(P<0.05)$.

Body weight gains (Fig. 3) differed greatly among diets ( $P<0.001$ by analysis of variance). Duncan's test showed that the negative diet differed from all others, as did the control diet, while there was no difference between the two supplemented diets.

The trends were similar for both males and females. However, on the three non-control diets, females tended to have lower weight gains and higher preference scores.

Both the weight gains and the behavioral measures showed large variation among individuals on each of the three non-control diets. Weight gains, for example, ranged from 200 to $610 \mathrm{~g} \mathrm{~d}^{-1}(\mathrm{SD}=118)$ on the negative diet, from 390 to $890 \mathrm{~g} \mathrm{~d}^{-1}(\mathrm{SD}=201)$ on the lysine-added, and from 480 to $950 \mathrm{~g} \mathrm{~d}^{-1}(\mathrm{SD}=$ 142) on the supplemented diet. These contrasted with the more uniform gains (range of $840-1020 \mathrm{~g} \mathrm{~d}^{-1}$, $\mathrm{SD}=63$ ) on the control diet. 
Fig. 3. Preference scores (indicating preferences for chewing the blood-impregnated tail model) in weeks 0 (pre-test), 2 and 4, and weight gain over the 4-wk test, for pigs fed the negative (N), lysine-added (L), supplemented (S) or control (C) diets in exp. 2. Each mean \pm SEM is based on $n=9$ or 10 pigs per treatment.

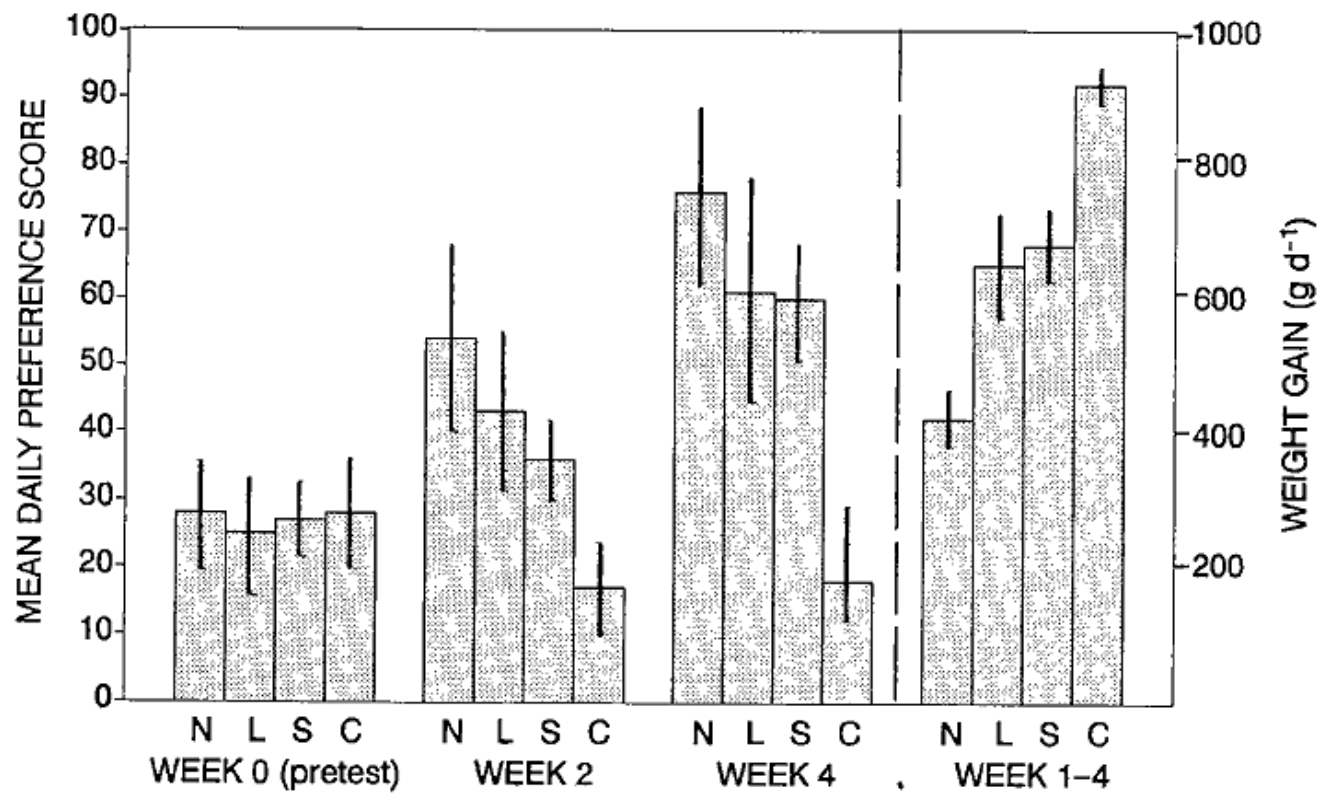

The differences in gain were related to the differences in attraction to blood (Fig. 2b). Several pigs on the lysine-added and supplemented diets had high weight gains similar to those of the controls. These pigs also had relatively low attraction to blood. Most of the pigs that developed the strongest response to the blood were animals with poor weight gains. However, there was much variation; in particular, some pigs with low weight gains failed to evidence enhanced attraction to blood.

Linear regression of preference scores in week 4 on weight gain (Fig. 2b) showed an overall slope of -0.11 ( \pm SE of $0.025, P<0.001$ ). The overall relationship is difficult to interpret because it involves a combination of variation within and between diets. Therefore, the relationship between preference score in week 4 and gain was calculated on a within-diet basis by expressing each pig's score and gain as deviations from the mean of all pigs on the same diet. This within-diet regression had a slope of $-0.10 \pm$ $0.041(P<0.02)$. Omission of the control diet (on the grounds that these animals were arguably not limited in gain by diet quality) made little difference to the result. Within each of the 3 deficient diets, the regression slopes were similar: $-0.10 \pm 0.113$ for the negative diet, $-0.11 \pm 0.086$ for the lysine-added diet, and $-0.10 \pm 0.067$ for the supplemented diet. In view of this relationship, the analysis of variance of preference scores was repeated with weight gain as a covariate. After adjustment for the covariate, differences attributable to diet did not approach statistical significance.

\section{DISCUSSION}

The first experiment showed that omission of soybean meal (the protein supplement) from a conventional growing pig ration caused a large increase in the animals' attraction to blood. The second experiment repeated this finding but failed to determine conclusively whether this effect was due simply to the poor amino acid composition of the diet.

The inconclusive result was due to two factors. First, the supplemented diet was intended to meet NRC recommendations in levels of essential amino acids and ideal protein; however, perhaps because of the formulation error, the diet supported substantially lower weight gains than the control. Hence, the attempt 
to fully replace the deficient amino acids with synthetic ones was not successful, and the two supplemented diets essentially constituted a single intermediate treatment. Second, although the use of supplemental amino acids caused a marked improvement in growth over the negative diet, the expected reduction in attraction to blood was not statistically significant. This is probably due in part to the very large individual variation in the behavioral measure.

The results suggest that there may be an underlying relationship between attraction to blood and dietary depression of weight gain. The combination of different diets and different individual responses to the diets produced a wide range of weight gains which were significantly related to attraction to blood. While this relationship involved both between- and within-diet effects, the similarity of the slopes was consistent with the hypothesis that a single underlying relationship between gain and attraction to blood could largely explain the findings. This is reinforced by the fact that differences between diets in attraction to blood became statistically nonsignificant when weight gain was included as a covariate in the analysis. Nonetheless, some pigs departed from the overall trend; in particular, a few slow-gaining pigs did not develop increased attraction to blood.

The relation between attraction to blood and tail-biting remains to be fully understood. We agree with the commonly-accepted view that genetic differences are relevant to tail-biting and that a wide variety of environmental social, and dietary factors can increase the likelihood of the behavior (e.g. Gadd 1967; Van Putten 1969; Fritschen and Hogg 1983; Sambraus 1985). Some of these factors presumably make the animals more restless and thus increase the amount or intensity of mutual biting and chewing that can lead to a bleeding injury. Once an injured tail is available in a group of pigs, we suspect that any heightened attraction to blood could promote an escalation of biting. Thus, we see attraction to blood contributing to tail-biting by causing the behavior to escalate once biting has begun (Fraser 1987b).

In this context, the present results suggest that diets whose protein supplementation is sufficiently poor to depress weight gains could increase the severity of tail-biting problems. Inadequate protein supplementation is commonly cited as a contributing factor in tail-biting (see Introduction) and these results suggest one mechanism whereby this might happen.

If our hypothesis is correct, it would be interesting to know how widely the link between depressed weight gain and heightened attraction to blood can be generalized. First, it seems unlikely that the increased attraction would be specific to blood alone. Perhaps a wide variety of flavors or chewable objects would become more attractive to an animal whose growth is depressed by inadequate protein supplementation (see Denton 1982) on "pica"). Second, the hypothesized link between depressed gains and attraction to blood may not be specific to diets with inadequate protein, but might apply also to depressed gains resulting from other dietary or environmental inadequacies. If so, this might help to explain why hunger (Sambraus 1985; Smith and Penny 1986) and many dietary deficiencies and environmental problems (Gadd 1967; Dalrymple 1978) have been claimed to increase the severity of tail-biting problems.

\section{ACKNOWLEDGMENTS}

We are indebted to Mr. R.A. Arcand who made most of the behavioral observations, and to the staff of the Animal Research Centre pig unit and feed mill for their helpful cooperation. Dr. B.K. Thompson kindly advised on the statistical analysis.

\section{REFERENCES}

Anderson, G. H. 1985. Proteins and amino acids: effects on the sympathetic nervous system and blood pressure regulation. Can. J. Physiol. Pharmacol. 64: 863-870. 
Anisman, H. and Zacharko, R. M. 1986. Behavioral and neurochemical consequences associated with stressors. Annal. N.Y. Acad. Sci. 467: 205-225.

Chamberlain, B., Ervin, E R., Pihl, R. O. and Young, S. N. 1987. The effect of raising or lowering tryptophan levels on aggression in vervet monkeys. Pharmacol. Biochem. Behav. 28: 503-510.

Dalrymple, J. R. 1978. Tail biting in swine. Factsheet 78-023 . Ontario Ministry of Agriculture and Food, Toronto, ON.

Denton, D. 1982. The hunger for salt. Springer-Verlag, Berlin, Germany

Fraser, D. 1987a. Attraction to blood as a factor in tail-biting by pigs. Appl. Anim. Behav. Sci. 17: 61-68.

Fraser, D. 1987b. Mineral-deficient diets and the pig's attraction to blood: Implications for tail-biting. Can. J. Anim. Sci. 67: 909-918.

Fritschen, R.D. and Hogg, A. 1983. Preventing tail biting in swine. NebGuide G75-246, revised. Institute of Agriculture and Natural Resources, Lincoln, NE.

Gadd, J. 1967. Tall biting. Pig Farming 15(7): 57-59.

Harper, A. E. and Peters, J. C. 1989. Protein intake, brain amino acid and serotonin concentrations and protein self-selection. J. Nutr. 119: 677-689.

Jericho, K. W. F. and Church, T.L. 1972. Cannibalism in pigs. Can. Vet. J. 13: 156-159.

Lacy, M.P., van Krey, H. P. and Denbow, D. M. 1984. Tyrosine and tryprophan influence on tonic immobility in the chicken. Poult. Sci. 63: 176-181.

Laycock, S. R. and Ball, R. O. 1990. Alleviation of hysteria in laying hens with dietary tryptophan. Can. J. Vet. Res. 54: 291-295.

Mench, J. A. and Shea, M. M. 1988. Effects of feed restriction and tryptophan on the behavior of broiler breeders. Proc. 1988 Maryland Nutr. Conf.: 40-46.

Munoz-Blanco, J. and Castillo, A. P. 1987. Changes in neurotransmitter amino acids content in several CNS areas from aggressive and nonaggressive bull strains. Physiol. Behav. 39: 453-457.

Peters, J. C. and Harper, A. E. 1985. Adaptation of rats to diets containing different levels of protein: effects on food intake, plasma and brain amino acid concentrations and brain neurotransmitter metabolism. J. Nutr. 115: 382-398.

Sambraus, H. H. 1985. Mouth-based anomalous syndromes. Pages 397-422 in A. F. Fraser, ed. Ethology of farm animals. Elsevier, Amsterdam, The Netherlands.

Smith, W. J. and Penny, R. H. C. 1986. Behavioral problems, including vices and cannibalism. Pages 762-772 in A. D. Leman et al. eds. Diseases of swine. 6th ed. Iowa State University Press, Ames, IA.

Van Putten, G. 1969. An investigation into tail-biting among fattening pigs. Br. Vet. J. 125: 511-517.

Williamson, D. H. 1987. Brain substrates and nutrition: symposium on nutrient metabolism and the central nervous system. Proc. Nutr. Soc. 46: 81-87. 